\title{
Granger-Causality in Quantiles between Financial Markets: Using Copula Approach*
}

\author{
Tae-Hwy Lee ${ }^{\dagger}$ \\ University of California, Riverside \\ Weiping Yang ${ }^{\ddagger}$ \\ Capital One Financial Research
}

May 2012

Revised: June 2013

\begin{abstract}
This paper considers the Granger-causality in conditional quantile and examines the potential of improving conditional quantile forecasting by accounting for such a causal relationship between financial markets. We consider Granger-causality in distributions by testing whether the copula function of a pair of two financial markets is the independent copula. Among returns on stock markets in the US, Japan and U.K., we find significant Granger-causality in distribution. For a pair of the financial markets where the dependent (conditional) copula is found, we invert the conditional copula to obtain the conditional quantiles. Dependence between returns of two financial markets is modeled using a parametric copula. Different copula functions are compared to test for Granger-causality in distribution and in quantiles. We find significant Granger-causality in the different quantiles of the conditional distributions between foreign stock markets and the US stock market. Granger-causality from foreign stock markets to the US stock market is more significant from UK than from Japan, while causality from the US stock market to UK and Japan stock markets is almost equally significant.
\end{abstract}

Keywords : Contagion in Financial Markets. Copula Functions. Inverting Conditional Copula. Granger-causality in Conditional Quantiles.

JEL Classification : C5

\footnotetext{
${ }^{*}$ We thank the editor Catherine Kyrtsou and two anonymous referees, Wolfgang Härdle, Yongmiao Hong, Peter Phillips, and the seminar participants at the Symposium on Econometric Theory and Applications (SETA) for useful comments. We also thank Yongmiao Hong for sharing his code used in Hong and Li (2005), which we modified for copula models in this paper. All errors are our own. A part of the research was started while Lee was visiting the California Institute of Technology. Lee thanks for their hospitality and the financial support during the visit. Yang thanks for the Chancellor's Distinguished Fellowship from the University of California, Riverside.

${ }^{\dagger}$ Corresponding author. Department of Economics, University of California, Riverside, CA 92521-0427, U.S.A. Tel: (951) 827-1509. Email: taelee@ucr.edu

${ }^{\ddagger}$ Capital One Financial Research, 15000 Capital One Drive, Richmond, VA 23233, U.S.A. Tel: (804) 284-6232. E-mail: weiping.yang@gmail.com
} 


\section{Introduction}

A causal relationship in a system of economic or financial time series has been widely studied. Following a series of seminal papers by Granger (1969, 1980 and 1988), Granger-causality (GC) test becomes a standard tool to detect causal relationship. Granger-causality in mean (GCM) is widely analyzed between macroeconomic variables, such as between money and income, consumption and output, etc. cf. Sims (1972, 1980), Stock and Watson (1989). In financial markets, a growing interest in volatility spill-over promotes the development of Granger-causality tests in volatility. cf. Granger, Robins and Engle (1986), Lin, Engle and Ito (1994), Cheung and Ng (1996), Comte and Liebermann (2000). Most tests of Granger-causality assume a bivariate Gaussian distribution and focus on Granger-causality in mean or variance.

A Gaussian distribution can not capture asymmetric dependence between financial markets. For instance, co-movements between different financial markets behave differently in a bull market and in a bear market. Ang and Chen (2002) assert that non-Gaussian dependence between economic variables or financial variables is prevalent. Associated with the non elliptical distribution, causality may matter in higher moments or in the dependence structure in a joint density. Thus, it is more informative to test Granger-causality in distribution (GCD) to explore a causal relationship between two financial time series.

We apply a copula-based approach to model the causality and dependence between a pair of two financial time series. Using copula density functions, we construct two tests for GCD. In the first test is nonparametric, following Hong and Li (2005), to compare the copula density in quadratic distance with the independent copula density. The second test is parametric, noting that

different parametric copula functions imply different dependence structure, we design a method to compare them in an entropy with the independent copula density. Both tests compare out-of-sample predictive ability of copula functions relative to the benchmark independent copula density.

GCD implies Granger-causality in some quantiles. In financial risk management and portfolio management, it is useful to know which quantiles leads to the GCD. In particular, Value-at-Risk $(\mathrm{VaR})$ is a quantile in tail that is widely used in capital budgeting and risk control. We are interested in exploring the potential of improving quantile forecasting of a trailing variable $Y$ using 
information of a preceding variable $X$. We define Granger-causality in quantile (GCQ), for which quantile forecasts are computed from inverting a conditional copula distribution, and we develop a test for GCQ.

In our empirical application, these copula-based methods are applied to analyze the pair-wise GCD from the Japan stock market to the US stock market (Japan-US), from the UK stock market to the US stock market (UK-US), from the US stock market to the Japan stock market (USJapan), and from the US stock market to the UK stock market (US-UK). We find significant GCD in these four data sets and all sample periods considered (seven different subsample periods), as the benchmark independent copula is clearly rejected in all data sets and subsamples. For GCQ, we compare predictive performance of various copula functions with the benchmark independent copula function over different quantiles of the conditional distribution of one market conditional on another market. It is found that GCQ is significant from US to foreign stock markets and from UK to the US stock market, but not from Japan to US. The result is robust over the seven subsamples.

The rest of the paper is organized as follows. Section 2 introduce two tests of GCD based on copula density functions. Both tests are based on the distance measures and thus measure the strength of GCD. Section 3 defines GCQ and develop a method to test for GCQ. Section 4 reports empirical findings on GCD and GCQ. Section 5 concludes. Section 6 is an appendix to review some basic results on copula functions.

\section{Granger-causality in Distribution}

In this section, we define GCD and introduce two statistics (based on the information entropy) which measure the strength of the GCD. The data used in our empirical applications are the daily return on the S\&P500 stock index (S\&P500), the NIKKEI 225 stock index (NIKKEI) and FTSE 100 stock index (FTSE). On the same trading day $t$, trading in the Tokyo Stock Exchange and London Stock Exchange precedes that in the New York Stock Exchange. To explore the causality between two financial markets, we use $\left\{X_{t}\right\}$ to denote the preceding variable and $\left\{Y_{t}\right\}$ as the trailing variable. For instance, $\left\{X_{t}\right\}$ denotes stock returns on the NIKKEI and $\left\{Y_{t}\right\}$ denotes stock returns on the S\&P500. See Table 1 (Panel A). We are only interested in causality in the same day or in the next day. Causality may occur in a longer time horizon. However, Dufour et al. (1998, 2006) 
shows that in the financial market, if there is non-causality between $X_{t}$ and $Y_{t}$, it will be difficult to explore Granger-causality in a longer horizon. With the development of information technology, impact of information in one market has the most significant effects in a short period, and we focus on causality in daily frequency.

Using a copula-based approach, various dependence structures can be flexibly modelled by a copula and marginal distribution functions. Dependence measures, such as Kendall's $\tau$ and Spearman's $\rho$, can also be easily computed using a copula function. Therefore, recently copula models have been widely used to model dependence between financial time series. Some recent research include Li (2000), Scaillet and Fermanian (2003), Embrechts et al. (2003), Patton (2006a,b), Granger et al. (2006), Chen and Fan (2006a,b), among others. We refer Appendix for more details. In this paper, we show how to use copula functions to test for GCD, how to measure the degree of GCD from using the log-likelihood of the copula density functions, and how to invert the conditional copula distribution functions to forecast the conditional quantiles which enable to test for GCQ.

We use the following notation. Let $R$ denote the sample size for estimation (for which we use a rolling scheme), $P$ the size of the out-of-sample period for forecast evaluation, and $T=R+P$. Suppose the stock market $X$ closes before the stock market $Y$ closes. Let $\mathcal{G}_{t}$ be the information set before the stock market $X$ closes and let $\mathcal{F}_{t}$ be the information set after the stock market $X$ closes but before the stock market $Y$ closes, i.e., $\mathcal{F}_{t}=\mathcal{G}_{t} \cup\left\{x_{t}\right\}$. Consider the conditional distribution functions, $F_{X}\left(x \mid \mathcal{G}_{t}\right)=\operatorname{Pr}\left(X_{t}<x \mid \mathcal{G}_{t}\right), F_{Y}\left(y \mid \mathcal{G}_{t}\right)=\operatorname{Pr}\left(Y_{t}<y \mid \mathcal{G}_{t}\right)$, and $F_{X Y}\left(x, y \mid \mathcal{G}_{t}\right)=\operatorname{Pr}\left(X_{t}<x\right.$ and $\left.Y_{t}<y \mid \mathcal{G}_{t}\right)$. Let $f_{X}\left(x \mid \mathcal{G}_{t}\right), f_{Y}\left(y \mid \mathcal{G}_{t}\right)$, and $f_{X Y}\left(x, y \mid \mathcal{G}_{t}\right)$ be the corresponding densities. Let $U_{t}=F_{X}\left(X_{t} \mid \mathcal{G}_{t}\right)$ and $V_{t}=F_{Y}\left(Y_{t} \mid \mathcal{G}_{t}\right)$ be the (conditional) probability integral transforms (PIT) of $X_{t}$ and $Y_{t}$. Let $C(u, v)$ and $c(u, v)$ be the conditional copula function and the conditional copula density function respectively. See Appendix for a brief introduction to the copula theory. We define GCD as follows.

Definition 1. (Non Granger-causality in distribution, NGCD): $\left\{X_{t}\right\}$ does not Grangercause $\left\{Y_{t}\right\}$ in distribution if and only if $\operatorname{Pr}\left(Y_{t}<y \mid \mathcal{F}_{t}\right)=\operatorname{Pr}\left(Y_{t}<y \mid \mathcal{G}_{t}\right)$ a.s. for all $y$.

There is GCD if $\operatorname{Pr}\left(Y_{t}<y \mid \mathcal{F}_{t}\right) \neq \operatorname{Pr}\left(Y_{t}<y \mid \mathcal{G}_{t}\right)$ for some $y .\left\{X_{t}\right\}$ does not Granger-cause $\left\{Y_{t}\right\}$ in distribution if $F_{Y}\left(y \mid \mathcal{F}_{t}\right)=F_{Y}\left(y \mid \mathcal{G}_{t}\right)$ a.s. This implies that testing for NGCD can be based on 
the null hypothesis

$$
H_{0}^{1}: f_{Y}\left(y \mid \mathcal{F}_{t}\right)=f_{Y}\left(y \mid \mathcal{G}_{t}\right)
$$

Note that the joint density is a product of the conditional density and the marginal density

$$
f_{X Y}\left(x, y \mid \mathcal{G}_{t}\right)=f_{Y}\left(y \mid \mathcal{F}_{t}\right) \times f_{X}\left(x \mid \mathcal{G}_{t}\right)
$$

and a joint density can be written from the decomposition theorem in (43) as

$$
f_{X Y}\left(x, y \mid \mathcal{G}_{t}\right)=f_{X}\left(x \mid \mathcal{G}_{t}\right) \times f_{Y}\left(y \mid \mathcal{G}_{t}\right) \times c(u, v) .
$$

From (2) and (3), we obtain

$$
f_{Y}\left(y \mid \mathcal{F}_{t}\right)=f_{Y}\left(y \mid \mathcal{G}_{t}\right) \times c(u, v) .
$$

Hence, the null hypothesis of NGCD, $H_{0}^{1}$ in (1), can be stated as the null hypothesis that the copula density is the independent copula,

$$
H_{0}^{2}: c(u, v)=1
$$

The test of GCD in (1) is equivalent to a test of measuring the distance between a copula density function conditional on $x_{t}$ and the independent copula.

We test for $H_{0}^{2}$ in $(5)$ by estimating $c(u, v)$ using a nonparametric predictive copula density

$$
\hat{c}_{P}(u, v)=\frac{1}{P} \sum_{t=R}^{T-1} K_{h}\left(u, \hat{u}_{t+1}\right) K_{h}\left(v, \hat{v}_{t+1}\right),
$$

where $K_{h}(\cdot)$ is a kernel function and

$$
\begin{aligned}
& \hat{u}_{t+1}=\hat{F}_{X}\left(x_{t+1}\right)=\frac{1}{R} \sum_{s=t-R+1}^{t} \mathbf{1}\left(x_{s} \leq x_{t+1}\right), \\
& \hat{v}_{t+1}=\hat{F}_{Y}\left(y_{t+1}\right)=\frac{1}{R} \sum_{s=t-R+1}^{t} \mathbf{1}\left(y_{s} \leq y_{t+1}\right),
\end{aligned}
$$

are the out-of-sample PIT values for $\left\{x_{t+1}\right\}_{t=R}^{T-1}$ and $\left\{y_{t+1}\right\}_{t=R}^{T-1}$ calculated with respect to the marginal empirical distribution functions $(\mathrm{EDF})$ that have been estimated using the rolling samples of the most recent $R$ observations at each time $t(=R, \ldots, T-1)$. To circumvent the boundary problem (as the PITs are bounded on [0 1]), we apply the boundary-modified kernel used by Hong and $\operatorname{Li}(2005)$ :

$$
K_{h}\left(a, a^{\prime}\right)=\left\{\begin{array}{lr}
h^{-1} k\left(\frac{a-a^{\prime}}{h}\right) / \int_{-(a / h)}^{1} k(u) d u, & \text { if } a \in[0, h), \\
h^{-1} k\left(\frac{a-a^{\prime}}{h}\right), & \text { if } a \in[h, 1-h), \\
h^{-1} k\left(\frac{a-a^{\prime}}{h}\right) / \int_{-1}^{(1-a) / h} k(u) d u, & \text { if } a \in(1-h, 1],
\end{array}\right.
$$


where $k(\cdot)$ is a symmetric kernel function and $h$ is the bandwidth.

For the null hypothesis $H_{0}^{2}$ in $(5)$, the test statistic is based on a quadratic form

$$
\hat{M}_{P}=\int_{0}^{1} \int_{0}^{1}\left[\hat{c}_{P}(u, v)-1\right]^{2} \mathrm{~d} u \mathrm{~d} v
$$

Instead of the quadratic distance between $\hat{c}_{P}(u, v)$ and the independent copula $c(u, v)=1$, the distance can be based on other measure such as Hellinger entropy. See Hong and Li (2005, footnote 12) on the comments on their test using the Hellinger entropy. See also Granger (2003, p. 695) for a similar but different statistic based on the Hellinger entropy between two densities. The statistic $\hat{M}_{P}$ based on the quadratic distance or based on the Hellinger entropy will provide a measure of the strength of GCD. The test statistic $\hat{M}_{P}$ is pivotalized by being centered and scaled as

$$
\hat{Q}_{P}=\left[P h \hat{M}_{P}-A_{h}^{0}\right] / V_{0}^{1 / 2}
$$

where $A_{h}^{0}$ is the nonstochastic centering factor and $V_{0}$ is the nonstochastic scale factor,

$$
\begin{aligned}
A_{h}^{0} & \equiv\left[\left(h^{-1}-2\right) \int_{-1}^{1} k^{2}(u) \mathrm{d} u+2 \int_{0}^{1} \int_{-1}^{b} k_{b}^{2}(u) \mathrm{d} u \mathrm{~d} b\right]^{2}-1, \\
V_{0} & \equiv 2\left[\int_{-1}^{1}\left[\int_{-1}^{1} k(u+v) k(v) \mathrm{d} v\right]^{2} \mathrm{~d} u\right]^{2}
\end{aligned}
$$

in which $k_{b}(\cdot)=k(\cdot) / \int_{-1}^{b} k(v) \mathrm{d} v$. Hong and Li (2005) show, under some regularity conditions, $\hat{Q}_{P}$ follows the standard normal distribution asymptotically as $P \rightarrow \infty$ under $H_{0}^{2}$ in (5). For the empirical analysis in Section $4, \hat{Q}_{P}$ and its asymptotic p-values are reported in Table 2. Larger $\hat{Q}_{P}$ and smaller p-values are evidence of stronger GCD.

As remarked above, while the statistic $\hat{Q}_{P}$ measures the distance between the nonparametric copula $\hat{c}_{P}(u, v)$ and the independent copula $c(u, v)=1$ using the quadratic distance in (10) or Hellinger entropy, we now consider another statistic based on the Kullback-Leibler (1951) information criterion (KLIC). KLIC is also known as the cross-entropy, relative entropy, or discrimination entropy. Rejection of the null hypothesis $H_{0}^{2}$ in (5) indicates that the copula is not the independent copula and there exists GCD. In such case, we want to model the GCD and measure the strength of the GCD. While this can be done by the nonparametric copula function $\hat{c}_{P}(u, v)$, parametric copula functions are widely used in the literature and in practice. Noting that there are numerous 
parametric copula functions, we now compare various parametric copula functions in their predictive ability (i.e., GCD). In the copula literature, most of the existing methods merely evaluate a parametric copula function - for instance using the Kolmogorov-Smirnov goodness of fit test, the $\chi^{2}$ goodness of fit test and the bivariate hit test. When we fail to reject more than one copula, we need a method to compare copula functions. We use the KLIC of a parametric copula density function $c_{k}$ relative to the true (unknown) copula function $c_{0}$

$$
K L I C\left(c_{k}\right)=\iint \log \left[c_{0}(u, v) / c_{k}(u, v)\right] c_{0}(u, v) \mathrm{d} u \mathrm{~d} v=E_{c_{0}}\left[\log c_{0}(u, v)-\log c_{k}(u, v)\right]
$$

A parametric copula density function $c_{k}$ is a better model if the KLIC distance is smaller. We use the word "distance" loosely because KLIC does not satisfy a triangle inequality. However, in this paper, as we will use the KLIC in comparing various competing parametric copula density models with a fixed benchmark copula model (i.e., the independent copula), the KLIC can serve as a distance metric with respect to the fixed benchmark. To compare copula model 1 (benchmark) and model $k(=2, \ldots, l)$, consider their KLIC-differential

$$
\begin{aligned}
D_{k} & =\operatorname{KLIC}\left(c_{1}\right)-\operatorname{KLIC}\left(c_{k}\right) \\
& =E_{c_{0}}\left[\log c_{0}(u, v)-\log c_{1}(u, v)\right]-E_{c_{0}}\left[\log c_{0}(u, v)-\log c_{k}(u, v)\right] \\
& =E_{c_{0}}\left[\log c_{k}(u, v)-\log c_{1}(u, v)\right]
\end{aligned}
$$

If $D_{k}>0, c_{k}$ is better than $c_{1}$ because $c_{k}$ is closer to $c_{0}$.

Taking model 1 (benchmark) as the independent copula with $\log c_{1}\left(u_{t}, v_{t}\right)=\log (1)=0$, we get

$$
D_{k}=E_{c_{0}}\left[\log c_{k}(u, v)\right]
$$

We estimate $D_{k}$ by

$$
\bar{D}_{k, P}=P^{-1} \sum_{t=R}^{T-1} \log c_{k}\left(\hat{u}_{t+1}, \hat{v}_{t+1}\right),
$$

which is the out-of-sample log-likelihood of the predictive copula density. The PITs $\left\{\hat{u}_{t+1}, \hat{v}_{t+1}\right\}$ are estimated as discussed above in (7) and (8). In the empirical section, $\bar{D}_{k, P}$ will be reported in Table 3. The copula density model $c_{k}(k=2, \ldots, l)$ with the largest value $\bar{D}_{k, P}$ will be preferred (which is shown in bold font in each row of Table 3). 
To statistically compare the copula functions $c_{k}(k=2, \ldots, l)$ and to test for GCD based on the multiple parametric copula functions, we consider the null hypothesis that none of the copula models $k=2, \ldots, l$ is better than the benchmark independent copula model $k=1$

$$
H_{0}^{3}: E\left(D_{k}\right) \leq 0, \quad k=2, \ldots, l
$$

Following White (2000), we consider the following null hypothesis

$$
H_{0}^{4}: \max _{k=2, \ldots, l} E\left(D_{k}\right) \leq 0
$$

under which the benchmark copula function is at least as good as all alternative copulas with regard to predictive ability. The test statistic for the null hypothesis $H_{0}^{4}$ is constructed as:

$$
\bar{V}_{l}=\max _{k=2, \ldots, l} P^{1 / 2} \bar{D}_{k, P}
$$

and its bootstrap distribution is obtained from

$$
\bar{V}_{l}^{*(b)}=\max _{k=2, \ldots, l} P^{1 / 2}\left(\bar{D}_{k, P}^{*(b)}-\bar{D}_{k, P}\right), b=1, \ldots, B
$$

where $\left\{\bar{D}_{k, P}^{*(b)}\right\}_{b=1}^{B}$ are computed from using stationary bootstrap of Politis and Romano (1994). Under some regularity conditions, the bootstrap distribution of $\bar{V}_{l}^{*(b)}$ approximates the true distribution of $\bar{V}_{l}$. In the empirical section, we have $l=7$ (seven copula models including the benchmark independent copula). The last column of Table 3 reports the bootstrap p-values to compare the benchmark independent copula with the other six copula models. A small p-value indicates that the null hypothesis of NGCD in (19) is rejected and that some parametric copula functions capture GCD.

\section{Granger-causality in Quantile}

Conditional quantile forecasting is more and more widely used in economic forecasting and finance. For example, A Value-at-Risk (VaR) is widely used in portfolio analysis and risk management. Our objective is to forecast the conditional quantile, $q_{\alpha}\left(Y_{t} \mid \mathcal{F}_{t}\right)$, where $\alpha$ is the left tail probability. The conditional quantile $q_{\alpha}\left(Y_{t} \mid \mathcal{F}_{t}\right)$ is derived from the inverse function of a conditional distribution function

$$
q_{\alpha}\left(Y_{t} \mid \mathcal{F}_{t}\right)=F_{Y}^{-1}\left(\alpha \mid \mathcal{F}_{t}\right),
$$


where $F_{Y}\left(y \mid \mathcal{F}_{t}\right)$ is the predicted conditional distribution function of $Y_{t}$. The inversion is to compute $q_{\alpha}\left(Y_{t} \mid \mathcal{F}_{t}\right)$ from

$$
\int_{-\infty}^{q_{\alpha}\left(y \mid \mathcal{F}_{t}\right)} f_{Y}\left(y \mid \mathcal{F}_{t}\right) \mathrm{d} y=\alpha
$$

where $f_{Y}\left(y \mid \mathcal{F}_{t}\right)$ is the predicted conditional density function. We now define GCQ for out-of-sample test.

Definition 2. (Non Granger-causality in quantile, NGCQ): $\left\{X_{t}\right\}$ does not Granger-cause $\left\{Y_{t}\right\}$ in $\alpha$-quantile if and only if $q_{\alpha}\left(Y_{t} \mid \mathcal{F}_{t}\right)=q_{\alpha}\left(Y_{t} \mid \mathcal{G}_{t}\right)$ a.s.

GC in conditional quantile refers to the case that $q_{\alpha}\left(Y_{t} \mid \mathcal{F}_{t}\right) \neq q_{\alpha}\left(Y_{t} \mid \mathcal{G}_{t}\right)$. If $X_{t}$ does not Grangercause $y_{t+1}$ in distribution, $q_{\alpha}\left(Y_{t} \mid \mathcal{F}_{t}\right)=q_{\alpha}\left(Y_{t} \mid \mathcal{G}_{t}\right)$ since $g_{t+1}\left(y \mid X_{t}, \mathcal{G}_{t}\right)=g_{t+1}\left(y \mid \mathcal{G}_{t}\right)$. Therefore, nonGranger-causality in distribution (NGCD) implies non-Granger-causality in conditional quantile (NGCQ). However, GCD does not necessarily imply GC in each quantile, while significant GC in any conditional quantile implies significant GC in distribution. For some quantiles, $X_{t}$ may Grangercause $Y_{t}$, while for other quantiles it may not. Granger (2003, p. 700) notes that some quantiles may differ from other quantiles in time series behavior (such as long memory and stationarity). For example, different parts of the distribution can have different time series properties; one tail could be stationary and the other tail may have a unit root. See also Granger (2010) for insightful vision of a pioneer on the importance of conditional quantiles.

The conditional quantiles can be obtained by an analytical approach, a historical approach or by a simulation-based approach. In practice an analytical approach using a parametric copula function is the most widely used approach, cf. Bouye and Salmon (2009). In this approach we set up quantitative models and derives close-form solutions for quantile forecasts as follows:

$$
\begin{aligned}
\operatorname{Pr}(Y \leq y \mid X=x) & =\lim _{\varepsilon \rightarrow 0} \operatorname{Pr}(Y \leq y \mid x \leq X \leq x+\varepsilon) \\
& =\lim _{\varepsilon \rightarrow 0} \frac{F_{X Y}(x+\varepsilon, y)-F_{X Y}(x, y)}{F_{X}(x+\varepsilon)-F_{X}(x)} \\
& =\lim _{\varepsilon \rightarrow 0} \frac{C\left(F_{X}(x+\varepsilon), F_{Y}(y)\right)-C\left(F_{X}(x), F_{Y}(y)\right)}{F_{X}(x+\varepsilon)-F_{X}(x)} \\
& =\frac{\partial}{\partial u} C(u, v),
\end{aligned}
$$

where $\frac{\partial}{\partial u} C(u, v)$ is the conditional copula distribution of $v$ given $u$. Denote $\frac{\partial}{\partial u} C(u, v)$ as $C_{u}(u, v)$. 
$q_{\alpha}\left(Y_{t} \mid \mathcal{F}_{t}\right)$ is computed by solving the equation

$$
C_{u}\left(F_{X}\left(x_{t+1}\right), F_{Y}\left(q_{\alpha}\left(Y_{t} \mid \mathcal{F}_{t}\right)\right)=\alpha\right.
$$

To evaluate predictive ability of those quantile forecasting models $q_{\alpha}\left(Y_{t} \mid \mathcal{F}_{t}\right)$ obtained from seven $(l=7)$ copula functions for $C(u, v)$, we use the "check" loss function of Koenker and Bassett (1978). The check loss function is a special case in a family of quasi-likelihood functions (Komunjer 2005), and can be used to measure the lack-of-fit of a quantile forecasting model. The expected check loss for a quantile forecast $q_{\alpha}\left(Y_{t} \mid \mathcal{F}_{t}\right)$ at a given $\alpha$ is

$$
Q(\alpha)=E\left[\alpha-\mathbf{1}\left(Y_{t}-q_{\alpha}\left(Y_{t} \mid \mathcal{F}_{t}\right)<0\right)\right]\left(Y_{t}-q_{\alpha}\left(Y_{t} \mid \mathcal{F}_{t}\right)\right)
$$

As seven copula functions are considered for $C(u, v)$, denote them as $C_{k}(u, v)(k=1, \ldots, l=7)$. For each copula distribution function $C_{k}(u, v)$, denote also the corresponding quantile forecast as $q_{\alpha, k}\left(Y_{t} \mid \mathcal{F}_{t}\right)$ and its expected check loss as $Q_{k}(\alpha)$. To compare copula model 1 (benchmark) and model $k(=2, \ldots, l)$, consider their check loss-differential

$$
D_{k}=Q_{1}(\alpha)-Q_{k}(\alpha)
$$

We estimate $D_{k}$ by

$$
\bar{D}_{k, P}=\hat{Q}_{1, P}(\alpha)-\hat{Q}_{k, P}(\alpha)
$$

where

$$
\hat{Q}_{k, P}(\alpha)=\frac{1}{P} \sum_{t=R}^{T-1}\left[\alpha-\mathbf{1}\left(Y_{t}-q_{\alpha}\left(Y_{t} \mid \mathcal{F}_{t}\right)<0\right)\right]\left(Y_{t}-q_{\alpha}\left(Y_{t} \mid \mathcal{F}_{t}\right)\right), \quad k=1, \ldots, l .
$$

The conditional quantile forecasts from using the copula distribution function $C_{k}(k=2, \ldots, l)$ with the largest value $\bar{D}_{k, P}$ will be preferred.

To statistically compare the conditional quantile forecast $q_{\alpha, k}\left(Y_{t} \mid \mathcal{F}_{t}\right)(k=2, \ldots, l)$ and to test for GCQ based on the multiple parametric copula functions, we consider the null hypothesis of NGCQ that none of the conditional quantile forecasts computed from copula $C_{k}(k=2, \ldots, l)$ is better than the benchmark quantile forecast computed from the independent copula distribution $C_{1}$

$$
H_{0}^{5}: E\left(D_{k}\right) \leq 0, \quad k=2, \ldots, l
$$


Following White (2000), we consider the following null hypothesis

$$
H_{0}^{6}: \max _{k=2, \ldots, l} E\left(D_{k}\right) \leq 0
$$

under which the benchmark quantile forecast is at least as good as all alternative conditional forecasts with regard to the check loss. The test statistic for the null hypothesis $H_{0}^{6}$ is constructed as:

$$
\bar{V}_{l}=\max _{k=2, \ldots, l} P^{1 / 2} \bar{D}_{k, P}
$$

The bootstrap p-value of this statistic is computed in the same way that we discussed for the KLIC based test for GCD in the previous section. Compute

$$
\bar{V}_{l}^{*(b)}=\max _{k=2, \ldots, l} P^{1 / 2}\left(\bar{D}_{k, P}^{*(b)}-\bar{D}_{k, P}\right), b=1, \ldots, B
$$

where $\left\{\bar{D}_{k, P}^{*(b)}\right\}_{b=1}^{B}$ are computed from using the stationary bootstrap of Politis and Romano (1994). Under some regularity conditions, the bootstrap distribution of $\bar{V}_{l}^{*(b)}$ approximates the true distribution of $\bar{V}_{l}$. In the empirical section, we have $l=7$ (seven copula models including the benchmark independent copula). The null hypothesis of NGCQ in (31) is that none of the six copula models (for GCQ) can produce better quantile forecasts than the Independent copula (for NGCQ). Each cell of Table 4 reports the bootstrap p-values to compare the benchmark quantile forecast with the other six quantile forecasts for a value of $\alpha$ and for a subsample. A small p-value indicates that the null hypothesis is rejected and that some parametric copula functions capture GCQ with better quantile forecast of $Y$ by conditioning on $X$.

\section{Empirical Analysis}

We investigate the causality and dependence between a pair of two financial markets using a copulabased approach. We focus on pair-wise causal relationships between three major stock markets of US, Japan, and UK. In particular, we compare multiple copula functions with Independent copula function, in order to test GCD and GCQ, of one market's return conditional on another market's return.

The data used in our empirical applications are the daily return on the S\&P500 stock index (S\&P500), the NIKKEI 225 stock index (NIKKEI) and FTSE 100 stock index (FTSE) from Jan. 3, 
1995 to Dec. 31, 2005. The source of the data is Yahoo Finance. To analyze the causal effects, we only keep the observations of the date when all three markets were open. The total observations in the data sets are 2566. Approximately, there are 230-240 observations per year and 20 observations per month.

On the same trading day, trading in the Tokyo Stock Exchange and London Stock Exchange precedes that in the New York Stock Exchange. To explore the causality among those financial markets, we use four data sets, which include stock returns on NIKKEI and S\&P500 (same day) (referred to as "Japan-US"), stock returns on FTSE and S\&P500 (same day) (referred to as "UKUS"), returns on S\&P500 and next day's NIKKEI (referred to as "US-Japan"), and returns on S\&P500 and next day's FTSE (referred to as "US-UK"), respectively.

To simplify the notation, in all four data sets, we use $\left\{X_{t}\right\}$ to denote the preceding variable and $\left\{Y_{t}\right\}$ as the trailing variable. For instance, in the data set of same day's stock returns on NIKKEI and S\&P500, $\left\{X_{t}\right\}$ denotes stock returns on the NIKKEI and $\left\{Y_{t}\right\}$ denotes stock returns on the S\&P500. Panel A of Table 1 reports detailed information of variables in those data sets. ${ }^{1}$

The focus of empirical study is out-of-sample forecasting. Hence, for each subsample, we choose 5 years (about $T=1170$ observations), in which 3 years data (about $R=700$ observations) are used for in-sample estimation of the models and 2 years data (about $P=470$ observations) are used for out-of-sample forecasting, of daily series. To conduct a robustness check and to explore the causality over time, the subsample shifts forward by 1 year. Therefore, there are altogether 7 subsamples. Details on the subsamples are listed in Panel B of Table 1.

In recent years, multivariate GARCH models are widely applied to model and forecast the temporal dependence and correlation between financial markets. Rather than assuming the bivariate normal distribution as in most multivariate models, we use a copula distribution. Because we are interested in testing GCD and GCQ instead of modeling the correlation between financial returns, we apply a relatively simple model in which the two univariate processes are modelled by a GARCH model and the dependence structure is modelled by a copula.

\footnotetext{
${ }^{1}$ Exchange rate fluctuations would influence stock market returns dominated by the US dollar in Japan and Britain. To remove the possible influence of such impacts, we also analyze causality between different financial markets using foreign exchange rate adjusted returns. We also study the same four data sets, but with USD dominated returns. The data source of the daily exchange rate of the Yen/USD and USD/GBP is the FERD database of Federal Reserve of St. Luis. As the results were basically the same, we do not report this FX-adjusted results but they are available upon request.
} 
Let $\left\{X_{t}\right\}$ and $\left\{Y_{t}\right\}$ be stock returns with zero mean (or demeaned). The estimation procedure is in two-steps following Chen and Fan (2006b). In the first step the univariate conditional variance for $\left\{X_{t}\right\}$ and $\left\{Y_{t}\right\}$ respectively is modeled by a univariate GARCH process, i.e.,

$$
\begin{aligned}
h_{x, t}^{-1 / 2} X_{t} & \sim N(0,1) \\
h_{x, t} & =\beta_{x 0}+\beta_{x 1} x_{t-1}^{2}+\beta_{x 2} h_{x, t-1}, \\
h_{y, t}^{-1 / 2} Y_{t} & \sim N(0,1) \\
h_{y, t} & =\beta_{y 0}+\beta_{y 1} y_{t-1}^{2}+\beta_{y 2} h_{y, t-1}
\end{aligned}
$$

In the second step the joint distribution is modeled by a copula function

$$
\left(h_{x, t}^{-1 / 2} X_{t}, h_{y, t}^{-1 / 2} Y_{t}\right) \sim C(u, v) .
$$

By choosing different copula functions and marginal distributions, this model can capture various dependence structure between financial time series. The model is estimated by a two-stage quasi maximum likelihood (QML) method. In the first stage, we estimate the two univariate GARCH models by QML method. With the estimated parameters in the GARCH model, the PIT values of the two marginal processes $\left\{u_{t}, v_{t}\right\}$ are estimated by the EDFs as in (7) and (8). In the second stage, we estimate the copula parameters following Chen and Fan (2006b).

\subsection{Granger-causality in Distribution}

We apply the proposed out-of-sample test for GCD following Hong and Li (2005). As discussed in Section 2 , we actually test $H_{0}: c(u, v)=1$. The forecasted conditional variance for $\left\{X_{t}\right\}$ and $\left\{Y_{t}\right\}$, $\hat{h}_{x, t+1}$ and $\hat{h}_{y, t+1}$, are computed by

$$
\begin{aligned}
& \hat{h}_{x, t+1}=\hat{\beta}_{x 0}+\hat{\beta}_{x 1} x_{t}^{2}+\hat{\beta}_{x 2} \hat{h}_{t, x}, \\
& \hat{h}_{y, t+1}=\hat{\beta}_{y 0}+\hat{\beta}_{y 1} y_{t}^{2}+\hat{\beta}_{y 2} \hat{h}_{t, y}
\end{aligned}
$$

Thus forecasted CDF values $\hat{u}_{t+1}$ and $\hat{v}_{t+1}$ for $x_{t+1}$ and $y_{t+1}$ are calculated by the empirical distribution function (EDF). A nonparametric copula function is estimated with paired EDF values

$\left\{\hat{u}_{t+1}, \hat{v}_{t+1}\right\}_{t=R}^{T-1}$ using product kernel functions. We use a quartic kernel in the boundary kernel function specified in Section 2, which is specified as

$$
k(u)=\frac{15}{16}\left(1-u^{2}\right)^{2} \mathbf{1}(|u| \leq 1) .
$$


For simplicity, bandwidths for $u$ and $v$ are assumed to be the same. Following Hong and Li (2005), we set $h=\hat{\sigma} R^{-1 / 6}$, where $\hat{\sigma}$ is the standard error of $\hat{u}_{t+1}$. This assumption can be relaxed and the results are not greatly affected.

In Table 2, we report the GCD results using the Hong and Li test statistic for $\left\{x_{t+1}, y_{t+1}\right\}_{t=R}^{T-1}$, computed by the method discussed in Section 2. We find significant GCD between all pairs of three financial markets. With the shift of subsamples, the GCD remains significant for all subsamples and data sets. Not only the foreign stock markets Granger-causes the US stock market, the information of the US market also affects the foreign markets in the following day. This finding is consistent with the literature on Granger-causality in variance between financial markets, e.g., Cheung and Ng (1996), Hong (2001), Sensier and van Dijk (2004).

The rejection of the null hypothesis that the copula is independent, $H_{0}: c(u, v)=1$, leads us to consider various parametric copula density functions. In the literature, Gaussian copulas, Frank copulas, Clayton copulas, Clayton Survival copulas and Gumbel copulas and Gumbel Survival copulas are the most commonly used copula functions. We use these copula functions (see Appendix). Specifically, we form the forecasted copula density function is $\hat{c}(u, v)$. Based on the decomposition theorem of a bivariate density in (43), the log-likelihood of a bivariate density is decomposed as

$$
\log f_{X Y}(x, y)=\log f_{X}(x)+\log f_{Y}(y)+\log c\left(F_{X}(x), F_{Y}(y)\right)
$$

Noting that we use the same marginal processes for all copula models, to compare predictive log-likelihood is equivalent to compare $\log \hat{c}\left(\hat{u}_{t+1}, \hat{v}_{t+1}\right)$, where $\hat{u}_{t+1}$ and $\hat{v}_{t+1}$ are computed using empirical distribution functions. Results are reported in Table 3. The independent copula is clearly rejected for all seven subsamples and all four data sets. This finding is consistent with the Hong-Li test results for GCD in Table 2 .

For Data Set 1A (Japan-US), the Clayton copula and the Gumbel Survival copula are the best two copulas, both of which exhibit increasing dependence in the lower tails. See Appendix on some properties of copulas. These two copula clear dominates the mirror counterparts, Clayton Survival copula and Gumbel copula, which have much smaller log-likelihood. The other data sets show similar pattern. In general, the best copula functions are Clayton or Gumbel Survival (both capture the left tail dependence). Even when the Gaussian copula is the best for some subsamples, 
Clayton or Gumbel Survival still dominate Clayton Survival or Gumbel in almost all cases (of the seven subsamples and four data sets).

This comparison of the copula only tells that left (lower) tail dependence is stronger than the right (upper) tail dependence. It does not mean that the upper tail dependence does not exist. In fact many cases show that the symmetric copula, namely Gaussian copula and the Frank copula are selected as the best copula function. The comparison of the copulas simply show that the bad news are generally more contagious than good news, but it does not show whether or not good news may Granger-cause the other market. In order to examine this, we need to examine the Granger-causality in various quantiles, GCQ, which we do next.

\subsection{Granger-causality in Quantiles}

As discussed in Section 3, significant GCD does not imply Granger-causality in each conditional quantile. In our empirical study, we focus on three regions of the distribution - the left tail (1\% quantile, $5 \%$ quantile and $10 \%$ quantile), the central region (40\% quantile, median and $60 \%$ quantile) and the right tail (90\% quantile, $95 \%$ quantile and $99 \%$ quantile). We compare the Granger-causality in those quantiles. To forecast the conditional quantile for $y_{t+1}$, because $x_{t+1}$ precedes $y_{t+1}, x_{t+1}$ is a realized value thus can be used in forecasting conditional quantile $q_{\alpha}\left(y_{t+1} \mid x_{t+1}\right)$. For instance, before the New York Stock Exchange opens, the Tokyo Stock Exchange has closed.

We compute the quantile forecasts by inverting the conditional copula distribution, as described in Section 3. We use six copulas - Gaussian, Frank, Clayton, Clayton Survival, Gumbel and Gumbel Survival copulas. These copula functions represent different dependence structures. We compare the check loss functions to evaluate predictive ability of different quantile forecasting copula models relative to the quantile forecasts without GCQ. ${ }^{2}$ The quantile forecasts without GCQ is computed from the marginal distribution of $y$, which is equivalent to the quantile forecast from using the Independent copula. The null hypothesis of the Reality Check test is that none of these six copula models (which models GCQ) makes better quantile forecast than the Independent copula (without GCQ). The results of testing GCQ in p-values are reported in Table 4. The small p-values of the

\footnotetext{
${ }^{2}$ We also use the loss functions proposed by Komunjer (2005) with $p=1,3$, and 5 respectively. Results using loss functions by Komunjer (2005) mimic those using a check loss function. Therefore, we only report results using a check loss function.
} 
Reality Check in Table 4 indicate the rejection of the null hypothesis, indicating that there exists a copula function to model GCQ and makes better quantile forecast of $Y$ by conditioning on $X$.

We report several interesting findings.

1. First, in every data set, a quantile forecasting model with no Granger-causality in quantile is rejected in many $\alpha$ quantiles, verifying the finding of significant GCD between financial markets. Meanwhile, for some regions, the quantile forecast model with non-Granger-causality can not be rejected. This in part verifies that GCD does not necessarily imply Grangercausality in every quantile.

2. The GCQ between US and Japan shows different patterns in Data Set 1A (Japan-US) than Data Set 1B (US-Japan). Data Set 1A (Japan-US) does not show many small p-values, indicating the Japanese stock market does not Granger-cause the US market in most quantiles. The Reality Check p-values for Data Set 1B (US-Japan) are very small across almost entire quantiles for all subsamples, indicating the US stock market strongly Granger-causes the Japanese stock market in all quantiles, left tail (bad performance), center (usual performance), and right tail (good performance) of the distribution of the NIKKEI returns conditional on the yesterday's S\&P500 returns. So there is clear asymmetry between US and Japan stock markets in GCQ.

3. It is interesting to see that the same asymmetry is not found between US and UK stock markets in GCQ. Looking at Panels $\mathrm{C}$ and D of Table 4, it is seen that there are small pvalues all over the places (across the quantiles and over different subsamples). GCQ is clear in both directions, from UK to US and also from US to UK markets, in all quantiles. GCQ is asymmetric between US and Japan financial markets, but more symmetric between US and UK markets. However, the results of Tables 2, 3, 4 suggest that the causality is even stronger from UK to US than from US to UK, although it is statistically significant in both directions.

\section{Conclusions}

Instead of testing Granger-causality in conditional mean or conditional variance, we consider testing for GCD by checking for independence in the copula function. Among the returns in three major 
stock markets, we find significant GCD. We also estimate several copula functions to model the GCD between financial markets, and invert the estimated conditional copula distribution function to obtained the conditional quantile functions, which enable us to examine the Granger-causality in various quantiles. We find that causality to the US stock market is more significant from UK than from Japan, while causality from the US stock market to UK and Japan stock markets is significant almost equally or slightly more to Japan than to UK.

The literature on GCQ is young and thin. The first paper on GCQ is Lee and Yang (2012), who explore money-income Granger causality in the conditional quantile and find that GCQ is significant in tail quantiles whereas it is not significant near the middle quantiles of the conditional distribution. Lee and Yang (2012) use the quantile regression to compute the out-of-sample quantile forecasts. While not reported in the paper for space, we have also computed the quantile forecasts from the quantile regressions instead of inverting the conditional copula functions. The results showed that the inverting conditional copula distribution is the superior approach to the quantile regressions especially towards both tails. Even in the middle of the distribution, the quantile regression was often dominated by the copula approach. This result was robust with all subsamples and data sets. This indicates that using a copula based approach has great potential to improve the quantile forecasting especially in the tails (such as VaRs) and perhaps even in the center of the distribution. Another advantage of inverting the conditional copula functions to obtain quantile forecasts instead of using the regression quantiles is that the former can avoid entirely the quantile-crossing problem while the latter methods requires some correction or adjustment as studied by Chernozhukov et al (2009). Jeong et al (2012) consider a nonparametric test for in-sample GCQ and examines the causal relations between the crude oil price, the USD/GBP exchange rate, and the gold price in the gold market.

The contribution of the current paper is two fold. First, we show how to test for GCD using the copula function and how to compare different parametric copula functions. These methods are based on quadratic distance or Hellinger entropy, and on the KLIC cross-entropy. The distance or entropy provides measures of the strength in GCD, as shown in Section 2. Second, we then show how to invert the predictive conditional copula functions to obtain conditional quantile forecasts and how to test for the out-of-sample GCQ, as shown in Section 3. Ashley, Granger and Schmalensee (1980) 
advocate that a test for Granger-causality of $X$ for $Y$ be conducted for out-of-sample predictive content of the conditioning variable $X$ for $Y$.

\section{Appendix: Copula}

In the framework of time series, a conditional copula is more often applied than a unconditional copula. As in Patton (2006b), we define the conditional copula as follows.

Sklar's Theorem for Conditional Copula: Let $F_{X Y}(x, y)$ be a bivariate conditional joint distribution function with conditional margin distributions $F_{X}(x)$ and $F_{Y}(y)$. Then there exists a conditional copula function $C$ such that for all $x, y$

$$
F_{X Y}(x, y)=C\left(F_{X}(x), F_{Y}(y)\right)
$$

where $F_{X}(x)=\operatorname{Pr}\left(X \leq x \mid \mathcal{F}_{t}\right), F_{Y}(y)=\operatorname{Pr}\left(Y \leq y \mid \mathcal{F}_{t}\right)$.

There are two important corollaries to this theorem:

Representation of Conditional Copula functions: The bivariate conditional copula function can be obtained from the bivariate conditional joint distribution function $F_{X Y}(x, y)$ by the following:

$$
C(u, v)=F_{X Y}\left(F_{X}^{-1}(u), F_{Y}^{-1}(v)\right)
$$

where $u=F_{X}(x)$ and $v=F_{Y}(y)$.

Decomposition of Bivariate Density: Let $f_{X Y}(x, y)=\frac{\partial^{2} F_{X Y}(x, y)}{\partial x \partial y}, f_{X}(x)=\frac{\partial F_{X}(x)}{\partial x}$, and $f_{Y}(y)=\frac{\partial F_{Y}(y)}{\partial y}$. Then

$$
f_{X Y}(x, y)=f_{X}(x) \times f_{Y}(y) \times c\left(F_{X}(x), F_{Y}(y)\right),
$$

where $c(u, v)=\frac{\partial^{2} C(u, v)}{\partial u \partial v}$ is the conditional copula density function.

Some parametric copula functions are widely used, such as Gaussian copula, Student- $t$ copula, Frank copula, Clayton copula, Gumbel copula, Gumbel Survival copula and Clayton survival copula. We use the following seven copula functions in this paper.

(1) Gaussian copula

$$
C^{\text {Gaussian }}(u, v, \rho)=\int_{-\infty}^{\Phi^{-1}(u)} \int_{-\infty}^{\Phi^{-1}(v)} \frac{1}{2 \pi \sqrt{1-\rho^{2}}} \exp \left(-\frac{r^{2}+s^{2}-2 \rho r s}{2\left(1-\rho^{2}\right)}\right) \mathrm{d} r \mathrm{~d} s, \rho \in(-1,1) .
$$


(2) Frank copula

$$
\begin{aligned}
C^{\text {Frank }}(u, v, \theta) & =-\frac{1}{\theta} \log \left(1+\frac{\left(e^{-\theta u}-1\right)\left(e^{-\theta v}-1\right)}{\left(e^{-\theta}-1\right)}\right), \theta \in(-\infty,+\infty) \backslash\{0\}, \\
c^{\text {Frank }}(u, v, \theta) & =\frac{-\theta\left(e^{-\theta}-1\right) e^{-\theta(u+v)}}{\left[\left(e^{-\theta}-1\right)+\left(e^{-\theta u}-1\right)\left(e^{-\theta v}-1\right)\right]^{2}}
\end{aligned}
$$

(3) Clayton copula

$$
\begin{aligned}
C^{\text {Clayton }}(u, v, \theta) & =\left(u^{-\theta}+v^{-\theta}-1\right)^{-1 / \theta}, \theta \in[-1,+\infty) \backslash\{0\}, \\
c^{\text {Clayton }}(u, v, \theta) & =\frac{(1+\theta)\left(u^{-\theta}+v^{-\theta}-1\right)^{-\frac{1}{\theta}-2}}{(u v)^{\theta+1}} .
\end{aligned}
$$

(4) Clayton Survival (CS) copula

$$
\begin{aligned}
C^{\mathrm{CS}}(u, v, \theta) & =u+v-1+\left((1-u)^{-\theta}+(1-v)^{-\theta}-1\right)^{-1 / \theta}, \theta \in[-1,+\infty) \backslash\{0\}, \\
c^{\mathrm{CS}}(u, v, \theta) & =c^{\text {Clayton }}(1-u, 1-v, \theta) .
\end{aligned}
$$

(5) Gumbel copula

$$
\begin{aligned}
C^{\text {Gumbel }}(u, v, \theta) & =\exp \left(-\left((-\log u)^{\theta}+(-\log v)^{\theta}\right)^{1 / \theta}\right), \theta \in[1,+\infty), \\
c^{\text {Gumbel }}(u, v, \theta) & =\frac{C^{\text {Gumbel }}(u, v, \theta)(\log u \log v)^{\theta-1}\left\{\left[(-\log u)^{\theta}+(-\log v)^{\theta}\right]^{1 / \theta}+\theta-1\right\}}{u_{1} u_{2}\left[(-\log u)^{\theta}+(-\log v)^{\theta}\right]^{2-1 / \theta}} .
\end{aligned}
$$

(6) Gumbel Survival (GS) copula

$$
\begin{aligned}
C^{\mathrm{GS}}(u, v, \theta) & =u+v-1+\exp \left\{-\left[(-\log (1-u))^{\theta}+(-\log (1-v))^{\theta}\right]^{1 / \theta}\right\}, \theta \in[1,+\infty), \\
c^{\mathrm{GS}}(u, v, \theta) & =c^{\mathrm{Gumbel}}(1-u, 1-v, \theta) .
\end{aligned}
$$

(7) Independent copula

$$
\begin{aligned}
C^{\operatorname{Indep}}(u, v) & =u v \\
c^{\operatorname{Indep}}(u, v) & =1 .
\end{aligned}
$$

Gaussian copula, $t$ copula and Frank copula are symmetric copula, while the others are asymmetric copula functions. Gumbel copula and CS copula demonstrate upper tail dependence, while GS copula and Clayton copula show lower tail dependence. With a Gaussian copula and two marginal Gaussian distribution, we can construct a bivariate Gaussian distribution. However, with non-Gaussian marginal distributions, even with a Gaussian copula, the constructed distribution will be non-Gaussian. 


\section{References}

Ang, A. and J. Chen (2002), "Asymmetric Correlations of Equity Portfolios", Review of Financial Studies 63, 443-494.

Ashley, R., C.W.J. Granger, and R. Schmalensee, (1980), "Advertising and Aggregate Consumption: An Analysis of Causality", Econometrica 48, 1149-1167.

Bouye, E. and M. Salmon (2009), "Copula Quantile Regressions and Tail Area Dynamic Dependence in Forex Markets," The European Journal of Finance 15, 721-750.

Chen, X. and Y. Fan (2005), "Pseudo-likelihood Ratio Tests for Semiparametric Multivariate Copula Model Selection", The Canadian Journal of Statistics 33(3), 389-414.

Chen, X. and Y. Fan (2006a), "Estimation of Copula-Based Semiparametric Time Series Models", Journal of Econometrics 130(2), 307-335.

Chen, X. and Y. Fan (2006b), "Estimation and Model Selection of Semiparametric Copula-based Multivariate Dynamic Models under Copula Misspecification" Journal of Econometrics 135, $125-154$.

Chernozhukov, V., I. Fernandez-Val and A. Galichon (2009), "Improving point and interval estimators of monotone functions by rearrangement," Biometrika 96(3), 559-575.

Cheung, Y.-W. and L.K. Ng (1996), "A Causality-in-Variance Test and its Application to Financial Market Prices", Journal of Econometrics 72, 33-48.

Comte, F. and O. Lieberman (2000), "Second Order Noncausality in Multivariate GARCH Processes", Journal of Time Series Analysis 21, 535-557.

Dufour, J. and E. Renault (1998), "Short Run and Long Run Causality in Time Series: Theory", Econometrica 66, 1099-1125.

Dufour, J., D. Pelletier and E. Renault (2006), "Short Run and Long Run Causality in Time Series: Inference", Journal of Econometrics 132, 337-362.

Embrechts, P., A. Hoing and A. Juri (2003), "Using Copulae to Bound the Value-at-Risk for Functions of Dependent Risks", Finance and Stochastics 7(2), 145-167.

Granger, C.W.J. (1969), "Investigating Causal Relations by econometric Models and CrossSpectral Methods", Econometrica 37, 424-438.

Granger, C.W.J. (1980), "Testing for Causality: A Personal Viewpoint", Journal of Economic Dynamics and Control 2, 329-352.

Granger, C.W.J. (1988), "Some Recent Developments in a Concept of Causality", Journal of Econometrics 39, 199-211. 
Granger, C.W.J. (2003), "Time Series Concepts for Conditional Distributions," Oxford Bulletin of Economics and Statistics 65, 689-701.

Granger, C.W.J. (2010), "Some Thoughts on the Development of Cointegration", Journal of Econometrics 158, 3-6.

Granger, C.W.J., R.P. Robins and R.F. Engle (1986). "Wholesale and Retail Prices: Bivariate Time Series Modelling with Forecastable Error Variances", in: D. Belsley and E. Kuh, eds., Model Reliability, MIT Press, Cambridge, MA, 117.

Granger, C.W.J., T. Teräsvitra and A. Patton (2006), "Common Factors in Conditional Distributions for Bivariate Time Series", Journal of Econometrics 132 (1), 43-57.

Hansen, P.R. (2005), "A Test for Superior Predictive Ability," Journal of Business and Economic Statistics 23, 365-380.

Hong, Y. (2001), "A Test for Volatility Spillover with Application to Exchange Rates", Journal of Econometrics 103, 183-224.

Hong, Y., and H. Li (2005), "Nonparametric Specification Testing for Continuous-Time Models with Applications to Term Structure of Interest Rates", Review of Financial Studies 18, 37-84.

Jeong, K., W.K. Härdle and S. Song (2012), "A Consistent Nonparametric Test for Causality in Quantile" Econometric Theory 28(4), 861-887.

Koenker, R and G. Basset (1978), "Asymptotic Theory of Least Absolute Error Regression", Journal of the American Statistical Association 73, 618-622.

Komunjer, I. (2005), "Quasi-Maximum Likelihood Estimation for Conditional Quantiles", Journal of Econometrics 128(1), 137-164.

Kullback, L. and R.A. Leibler (1951), "On Information and Sufficiency", Annals of Mathematical Statistics 22, 79-86.

Lee, T.-H. and W. Yang (2012), "Money-Income Granger-Causality in Quantiles", Advances in Econometrics, Volume 30, Chapter 12, pp. 383-407, Millimet, D. and Terrell, D. (ed.), Emerald Publishers.

Li, D.X. (2000), "On Default Correlation: A Copula Function Approach", Journal of Fixed Income $9(4), 43-54$.

Lin, W.L., R.F. Engle and T. Ito (1994), Do Bulls and Bears Move Across Borders? International transmission stock returns and volatility, Review of Financial Studies 7, 507-538.

Patton, A.J. (2006a), "Estimation of Multivariate Models for Time Series of Possibly Different Lengths", Journal of Applied Econometrics 21(2), 147-173. 
Patton, A.J. (2006b), "Modelling Asymmetric Exchange Rate Dependence", International Economic Review 47(2), 527-556.

Politis, D.N. and J.P. Romano (1994), "The Stationary Bootstrap", Journal of the American Statistical Association 89, 1303-1313.

Scaillet, O. and J.D. Fermanian (2003), "Nonparametric Estimation of Copulas for Time Series", Journal of Risk 5, 25-54.

Sensier, M. and D. van Dijk (2004), "Testing for Volatility Changes in US Macroeconomic Time Series", Review of Economics and Statistics 86, 833-839.

Sims, C.A. (1972), "Money, Income and Causality", American Economic Review 62, 540-542.

Sims, C.A. (1980), "Comparison of Interwar and Postwar Business Cycles: Monetarism Reconsidered", American Economic Review 70, 250-259.

Stock, J.H. and M.W. Watson (1989), "Interpreting the Evidence on Money-Income Causality", Journal of Econometrics 40, 161-181.

White, H. (2000), “A Reality Check for Data Snooping”, Econometrica 68, 1097-1126. 
Table 1. Description of Data Sets and Subsamples

\begin{tabular}{|l|l|l|c|}
\hline & \multicolumn{1}{|c|}{ P } & \multicolumn{1}{|c|}{$Y$} & Observations \\
\hline Data Set 1A (Japan-US) & returns on NIKKEI 225 & returns on S\&P500 & 2566 \\
Data Set 1B (US-Japan) & returns on S\&P500 & returns on NIKKEI 225 for next day & 2566 \\
Data Set 2A (UK-US) & returns on FTSE 100 & returns on S\&P501 & 2566 \\
Data Set 2B (US-UK) & returns on S\&P500 & returns on FTSE 100 for next day & 2566 \\
\hline
\end{tabular}

Panel B. Subsamples in Each Date Set

\begin{tabular}{|c|c|c|c|c|c|}
\hline & Starting Date & Ending Date & $T$ & $R$ & $P$ \\
\hline Subsample 1 & Jan-95 & Dec-99 & 1172 & 706 & 466 \\
Subsample 2 & Jan-96 & Dec-00 & 1171 & 702 & 469 \\
Subsample 3 & Jan-97 & Dec-01 & 1164 & 699 & 465 \\
Subsample 4 & Jan-98 & Dec-02 & 1163 & 703 & 460 \\
Subsample 5 & Jan-99 & Dec-03 & 1162 & 697 & 465 \\
Subsample 6 & Jan-00 & Dec-04 & 1162 & 697 & 465 \\
Subsample 7 & Jan-01 & Dec-05 & 1157 & 693 & 464 \\
\hline
\end{tabular}


Table 2. Testing for GCD

\begin{tabular}{|l|c|r|c|c|}
\hline & Data Set 1A & \multicolumn{1}{|c|}{ Data Set 1B } & Data Set 2A & Data Set 2B \\
\hline Subsample 1 & $3.68(0.000)$ & $4.59(0.000)$ & $15.47(0.000)$ & $7.46(0.000)$ \\
Subsample 2 & $1.68(0.093)$ & $10.11(0.000)$ & $12.80(0.000)$ & $5.14(0.000)$ \\
Subsample 3 & $0.71(0.478)$ & $11.57(0.000)$ & $18.51(0.000)$ & $4.64(0.000)$ \\
Subsample 4 & $2.07(0.039)$ & $11.46(0.000)$ & $24.70(0.000)$ & $7.25(0.000)$ \\
Subsample 5 & $2.83(0.005)$ & $13.16(0.000)$ & $15.63(0.000)$ & $4.97(0.000)$ \\
Subsample 6 & $4.41(0.000)$ & $12.50(0.000)$ & $13.53(0.000)$ & $4.36(0.000)$ \\
Subsample 7 & $3.82(0.000)$ & $11.53(0.000)$ & $12.01(0.000)$ & $5.98(0.000)$ \\
\hline
\end{tabular}

Notes: Reported are the Hong and Li (2005) test statistics for the null hypothesis of non-GCD. The null hypothesis is that the copula density function is the independent copula, i.e., $c(u, v)=1$. The test is an out-of-sample test. The asymptotic p-values calculated from the standard normal distribution are shown in brackets. 
Table 3. Testing for GCD and Comparing Parametric Copula Functions

Panel A. Data Set 1A (Japan-US)

\begin{tabular}{|l|c|c|c|c|c|c|c|}
\hline & Gaussian & Frank & Clayton & $\begin{array}{c}\text { Clayton } \\
\text { Survival }\end{array}$ & Gumbel & $\begin{array}{c}\text { Gumbel } \\
\text { Survival }\end{array}$ & P-value \\
\hline Subsample 1 & 0.0094 & 0.0082 & $\mathbf{0 . 0 1 3 0}$ & 0.0042 & 0.0081 & 0.0121 & 0.007 \\
Subsample 2 & 0.0036 & 0.0036 & $\mathbf{0 . 0 0 4 7}$ & -0.0006 & -0.0007 & 0.0032 & 0.327 \\
Subsample 3 & 0.0110 & 0.0113 & 0.0124 & 0.0050 & 0.0070 & $\mathbf{0 . 0 1 5 3}$ & 0.058 \\
Subsample 4 & 0.0255 & 0.0245 & 0.0308 & 0.0145 & 0.0199 & $\mathbf{0 . 0 3 5 3}$ & 0.000 \\
Subsample 5 & 0.0151 & 0.0162 & 0.0175 & 0.0082 & 0.0107 & $\mathbf{0 . 0 1 7 6}$ & 0.025 \\
Subsample 6 & 0.0106 & $\mathbf{0 . 0 1 0 8}$ & 0.0079 & 0.0061 & 0.0069 & 0.0065 & 0.096 \\
Subsample 7 & $\mathbf{0 . 0 0 7 7}$ & 0.0065 & 0.0034 & 0.0049 & 0.0055 & 0.0040 & 0.272 \\
\hline
\end{tabular}

Panel B. Data Set 1B (US-Japan)

\begin{tabular}{|l|c|c|c|c|c|c|c|}
\hline & & & & Clayton & & Gumbel & Survival \\
P-value \\
\hline Subsample 1 & 0.0424 & 0.0341 & 0.0429 & 0.0240 & 0.0310 & $\mathbf{0 . 0 4 3 7}$ & 0.002 \\
Subsample 2 & $\mathbf{0 . 0 8 9 2}$ & 0.0739 & 0.0756 & 0.0616 & 0.0729 & 0.0858 & 0.000 \\
Subsample 3 & $\mathbf{0 . 0 7 5 6}$ & 0.0685 & 0.0633 & 0.0475 & 0.0554 & 0.0716 & 0.000 \\
Subsample 4 & $\mathbf{0 . 0 6 3 8}$ & 0.0636 & 0.0445 & 0.0478 & 0.0547 & 0.0536 & 0.000 \\
Subsample 5 & $\mathbf{0 . 0 9 5 0}$ & 0.0912 & 0.0636 & 0.0748 & 0.0829 & 0.0742 & 0.000 \\
Subsample 6 & $\mathbf{0 . 1 0 6 8}$ & 0.0982 & 0.0749 & 0.0791 & 0.0908 & 0.0840 & 0.000 \\
Subsample 7 & $\mathbf{0 . 0 8 9 1}$ & 0.0763 & 0.0715 & 0.0579 & 0.0734 & 0.0774 & 0.000 \\
\hline
\end{tabular}

Panel C. Data Set 2A (UK-US)

\begin{tabular}{|l|c|c|c|c|c|c|c|}
\hline & Gaussian & Frank & Clayton & $\begin{array}{c}\text { Clayton } \\
\text { Survival }\end{array}$ & Gumbel & $\begin{array}{c}\text { Gumbel } \\
\text { Survival }\end{array}$ & P-value \\
\hline Subsample 1 & 0.1198 & 0.1035 & 0.1095 & 0.0826 & 0.1044 & $\mathbf{0 . 1 2 2 5}$ & 0.000 \\
Subsample 2 & 0.1054 & 0.0904 & 0.1002 & 0.0685 & 0.0874 & $\mathbf{0 . 1 1 1 3}$ & 0.000 \\
Subsample 3 & $\mathbf{0 . 1 3 6 9}$ & 0.1273 & 0.1135 & 0.0976 & 0.1228 & 0.1283 & 0.000 \\
Subsample 4 & $\mathbf{0 . 1 6 9 8}$ & 0.1501 & 0.1289 & 0.1387 & 0.1658 & 0.1564 & 0.000 \\
Subsample 5 & $\mathbf{0 . 1 3 9 1}$ & 0.1133 & 0.1021 & 0.1235 & 0.1371 & 0.1285 & 0.000 \\
Subsample 6 & 0.0937 & 0.0861 & 0.0581 & 0.0937 & $\mathbf{0 . 1 0 1 4}$ & 0.0848 & 0.000 \\
Subsample 7 & 0.0728 & 0.0686 & 0.0324 & 0.0947 & $\mathbf{0 . 0 9 8 7}$ & 0.0603 & 0.000 \\
\hline
\end{tabular}

Panel D. Data Set 2B (US-UK)

\begin{tabular}{|l|c|c|c|c|c|c|c|}
\hline & Gaussian & Frank & Clayton & $\begin{array}{c}\text { Clayton } \\
\text { Survival }\end{array}$ & Gumbel & $\begin{array}{c}\text { Gumbel } \\
\text { Survival }\end{array}$ & P-value \\
\hline Subsample 1 & 0.0436 & 0.0376 & $\mathbf{0 . 0 5 3 5}$ & 0.0166 & 0.0254 & 0.0523 & 0.000 \\
Subsample 2 & 0.0452 & $\mathbf{0 . 0 4 7 1}$ & 0.0422 & 0.0230 & 0.0274 & 0.0444 & 0.000 \\
Subsample 3 & $\mathbf{0 . 0 4 3 3}$ & 0.0402 & 0.0421 & 0.0193 & 0.0256 & 0.0408 & 0.002 \\
Subsample 4 & 0.0360 & $\mathbf{0 . 0 3 7 1}$ & 0.0349 & 0.0187 & 0.0263 & 0.0358 & 0.013 \\
Subsample 5 & 0.0356 & $\mathbf{0 . 0 3 8 8}$ & 0.0270 & 0.0253 & 0.0288 & 0.0325 & 0.005 \\
Subsample 6 & 0.0383 & 0.0312 & $\mathbf{0 . 0 4 6 7}$ & 0.0123 & 0.0184 & 0.0452 & 0.001 \\
Subsample 7 & 0.0398 & 0.0308 & $\mathbf{0 . 0 6 5 7}$ & 0.0051 & 0.0176 & 0.0571 & 0.000 \\
\hline
\end{tabular}

Notes: Reported are the out-of-sample average of the logarithm of the predictive copula density function, log $c(u, v)$ for each copula model for each subsample. For the independent copula, $\log c(u, v)$ is zero. Positive values of the out-of-sample average of the logarithm of the predictive copula density function indicate the presence of GCD. The larger the value is, the better is the copula function. The largest value in each row is shown in bold font to indicate the best copula model. To statistically compare these copula functions, we take the independence copula (non-GCD) as the benchmark and compare it with other copula functions. The last column reports the Reality-Check (White 2000) p-values to compare the 6 copula models with the benchmark independent copula. P-values reported are Hansen's p-values (Hansen 2005). The null hypothesis of the reality check test is that none of the six copula functions is better than the independent copula. Almost all p-values are very small, rejecting the null hypothesis of no-GCD, indicating that some parametric copula functions capture GCD. 
Table 4. Testing for GCQ

Panel A. Data Set 1A (Japan-US)

\begin{tabular}{|l|c|c|c|c|c|c|c|c|c|c|c|c|c|}
\hline & $1 \%$ & $5 \%$ & $10 \%$ & $20 \%$ & $30 \%$ & $40 \%$ & $50 \%$ & $60 \%$ & $70 \%$ & $80 \%$ & $90 \%$ & $95 \%$ & $99 \%$ \\
\hline Subsample 1 & 0.285 & 0.314 & 0.205 & 0.098 & 0.054 & 0.124 & 0.105 & 0.313 & 0.344 & 0.406 & 0.407 & 0.144 & 0.233 \\
Subsample 2 & 0.041 & 0.610 & 0.308 & 0.190 & 0.321 & 0.474 & 0.775 & 0.833 & 0.928 & 0.824 & 0.920 & 0.789 & 0.442 \\
Subsample 3 & 0.091 & 0.707 & 0.581 & 0.440 & 0.404 & 0.373 & 0.422 & 0.437 & 0.479 & 0.357 & 0.802 & 0.482 & 0.821 \\
Subsample 4 & 0.310 & 0.227 & 0.112 & 0.266 & 0.257 & 0.053 & 0.372 & 0.368 & 0.407 & 0.320 & 0.209 & 0.137 & 0.699 \\
Subsample 5 & 0.187 & 0.264 & 0.170 & 0.527 & 0.060 & 0.037 & 0.015 & 0.206 & 0.491 & 0.724 & 0.493 & 0.636 & 0.793 \\
Subsample 6 & 0.093 & 0.020 & 0.006 & 0.025 & 0.078 & 0.052 & 0.122 & 0.672 & 0.788 & 0.784 & 0.291 & 0.573 & 0.449 \\
Subsample 7 & 0.534 & 0.421 & 0.315 & 0.021 & 0.181 & 0.276 & 0.772 & 0.824 & 0.777 & 0.460 & 0.554 & 0.621 & 0.061 \\
\hline
\end{tabular}

Panel B. Data Set 1B (US-Japan)

\begin{tabular}{|l|c|c|c|c|c|c|c|c|c|c|c|c|c|}
\hline & $1 \%$ & $5 \%$ & $10 \%$ & $20 \%$ & $30 \%$ & $40 \%$ & $50 \%$ & $60 \%$ & $70 \%$ & $80 \%$ & $90 \%$ & $95 \%$ & $99 \%$ \\
\hline Subsample 1 & 0.010 & 0.213 & 0.165 & 0.059 & 0.022 & 0.030 & 0.133 & 0.129 & 0.147 & 0.279 & 0.197 & 0.323 & 0.533 \\
Subsample 2 & 0.099 & 0.000 & 0.002 & 0.000 & 0.000 & 0.000 & 0.000 & 0.000 & 0.004 & 0.044 & 0.266 & 0.323 & 0.211 \\
Subsample 3 & 0.337 & 0.010 & 0.001 & 0.002 & 0.001 & 0.000 & 0.000 & 0.000 & 0.017 & 0.029 & 0.062 & 0.171 & 0.020 \\
Subsample 4 & 0.570 & 0.103 & 0.005 & 0.000 & 0.000 & 0.000 & 0.003 & 0.018 & 0.032 & 0.031 & 0.068 & 0.009 & 0.052 \\
Subsample 5 & 0.269 & 0.016 & 0.000 & 0.000 & 0.000 & 0.001 & 0.001 & 0.001 & 0.000 & 0.000 & 0.002 & 0.001 & 0.239 \\
Subsample 6 & 0.097 & 0.001 & 0.000 & 0.000 & 0.000 & 0.000 & 0.000 & 0.000 & 0.000 & 0.000 & 0.001 & 0.007 & 0.156 \\
Subsample 7 & 0.086 & 0.007 & 0.000 & 0.000 & 0.000 & 0.007 & 0.001 & 0.001 & 0.005 & 0.010 & 0.033 & 0.025 & 0.060 \\
\hline
\end{tabular}

Panel C. Data Set 2A (UK-US)

\begin{tabular}{|l|c|c|c|c|c|c|c|c|c|c|c|c|c|}
\hline & $1 \%$ & $5 \%$ & $10 \%$ & $20 \%$ & $30 \%$ & $40 \%$ & $50 \%$ & $60 \%$ & $70 \%$ & $80 \%$ & $90 \%$ & $95 \%$ & $99 \%$ \\
\hline Subsample 1 & 0.040 & 0.373 & 0.352 & 0.276 & 0.155 & 0.079 & 0.006 & 0.006 & 0.016 & 0.019 & 0.039 & 0.054 & 0.032 \\
Subsample 2 & 0.091 & 0.004 & 0.003 & 0.002 & 0.000 & 0.000 & 0.000 & 0.000 & 0.000 & 0.002 & 0.013 & 0.046 & 0.072 \\
Subsample 3 & 0.043 & 0.005 & 0.000 & 0.000 & 0.000 & 0.000 & 0.000 & 0.000 & 0.000 & 0.000 & 0.012 & 0.138 & 0.408 \\
Subsample 4 & 0.376 & 0.339 & 0.193 & 0.001 & 0.000 & 0.000 & 0.000 & 0.000 & 0.000 & 0.000 & 0.001 & 0.009 & 0.122 \\
Subsample 5 & 0.438 & 0.389 & 0.332 & 0.193 & 0.003 & 0.000 & 0.000 & 0.000 & 0.000 & 0.000 & 0.000 & 0.002 & 0.373 \\
Subsample 6 & 0.421 & 0.174 & 0.167 & 0.008 & 0.000 & 0.002 & 0.002 & 0.011 & 0.008 & 0.002 & 0.005 & 0.014 & 0.065 \\
Subsample 7 & 0.751 & 0.176 & 0.082 & 0.009 & 0.005 & 0.012 & 0.012 & 0.006 & 0.000 & 0.000 & 0.001 & 0.004 & 0.392 \\
\hline
\end{tabular}

Panel D. Data Set 2B (US-UK)

\begin{tabular}{|l|c|c|c|c|c|c|c|c|c|c|c|c|c|}
\hline & $1 \%$ & $5 \%$ & $10 \%$ & $20 \%$ & $30 \%$ & $40 \%$ & $50 \%$ & $60 \%$ & $70 \%$ & $80 \%$ & $90 \%$ & $95 \%$ & $99 \%$ \\
\hline Subsample 1 & 0.104 & 0.236 & 0.040 & 0.005 & 0.001 & 0.001 & 0.001 & 0.016 & 0.039 & 0.080 & 0.417 & 0.813 & 0.613 \\
Subsample 2 & 0.588 & 0.194 & 0.053 & 0.008 & 0.009 & 0.003 & 0.003 & 0.008 & 0.005 & 0.012 & 0.188 & 0.690 & 0.796 \\
Subsample 3 & 0.281 & 0.188 & 0.019 & 0.000 & 0.005 & 0.020 & 0.016 & 0.035 & 0.043 & 0.059 & 0.082 & 0.209 & 0.369 \\
Subsample 4 & 0.225 & 0.032 & 0.016 & 0.021 & 0.030 & 0.056 & 0.055 & 0.098 & 0.117 & 0.055 & 0.104 & 0.172 & 0.751 \\
Subsample 5 & 0.416 & 0.039 & 0.137 & 0.046 & 0.065 & 0.042 & 0.051 & 0.087 & 0.093 & 0.071 & 0.054 & 0.077 & 0.552 \\
Subsample 6 & 0.051 & 0.065 & 0.031 & 0.024 & 0.021 & 0.011 & 0.014 & 0.209 & 0.343 & 0.233 & 0.137 & 0.183 & 0.502 \\
Subsample 7 & 0.048 & 0.000 & 0.000 & 0.004 & 0.002 & 0.001 & 0.000 & 0.012 & 0.115 & 0.070 & 0.135 & 0.517 & 0.741 \\
\hline
\end{tabular}

Notes: We compute the quantile forecasts by inverting the parametric conditional copula distribution. We use six copulas (Gaussian, Frank, Clayton, Clayton Survival, Gumbel and Gumbel Survival copulas). The check loss functions is compared to evaluate predictive ability of different quantile forecasting using different copula models. The benchmark quantile forecasts are computed using the independent copula, so that there is no GCQ. Reported are the bootstrap p-values for testing the null hypothesis that none of these six copula models (which models GCQ) makes better quantile forecast than the Independent copula (which gives no GCQ). The small pvalues of the Reality Check indicate the rejection of the null hypothesis, indicating that there exists a copula function to model GCQ and makes better quantile forecast. 